Revista Temas Socio Jurídicos

Vol. 38 N $^{\circ} 76$ Enero - Junio de 2019

ISSN: 0120-8578

ISSN electrónico: 2590-8901

\title{
PROBLEMÁTICA LABORAL EN LOS CENTROS GERONTOLÓGICOS EN LA CIUDAD DE MEDELLÍN
}

Juan Guillermo Espinal*

Recibido: Febrero 26 de 2019

Aprobado: Abril 30 de 2019

\begin{abstract}
RESUMEN:
Los centros gerontológicos, en un gran porcentaje, están constituidos como Entidades Sin Ánimo de Lucro (ESAL), cuya naturaleza es desarrollar su objeto social bajo el criterio de la prestación altruista de servicios básicos a los adultos mayores, con el fin de que estos adultos logren vivir sus últimos años en condiciones de dignidad, en lugar de favorecer el lucro personal de quienes desarrollan la actividad. Este aspecto de obrar sin ánimo de lucro, lleva a que una gran mayoría de estos centros no cuenten con los recursos suficientes para desarrollar sus actividades, de tal manera que sean cubiertas todas las necesidades que tienen los usuarios. Esta situación conduce a que muchas de las funciones que deben cumplir los profesionales y expertos que tienen a su cargo la atención y el cuidado de los adultos mayores no tengan una clara separación de sus funciones ni tampoco que sobre ellos se aplique una contratación laboral adecuada, ya que muchas veces se superponen sus funciones con las de otros auxiliares, trayendo como consecuencia una prestación del servicio inadecuada. Del mismo modo que la contratación por horas, por prestación de servicios o por labor, les niega una estabilidad laboral, esto obliga
\end{abstract}

Citar este trabajo como: Guillermo Espinal, J. (2019). Problemática laboral en los centros gerontológicos en la ciudad de Medellín. Temas Socio-Jurídicos, 38(76), pp. 54-80. https://doi.org/ 10.29375/01208578.3579

* Abogado de la Universidad de Medellín. Magíster en Historia de la Universidad de Antioquia. Docente investigador del grupo de investigación Orbis Iuris, Facultad de Derecho, Fundación Universitaria Autónoma de las Américas sede Medellín. Orcid: https:// orcid.org/0000-0001-8555-4253. Correo electrónico: juan.espinal@uam.edu.co 
a que acudan a contratar, paralelamente, con otras entidades o a dedicar mucho de su tiempo en otras actividades, con lo cual se pierde no solo la especialización en los servicios ofertados sino el sentido humano de un cuidado permanente de los usuarios. Lo anterior se convierte en un obstáculo más para brindar una adecuada prestación de los servicios y para la estabilidad y permanencia de los centros gerontológicos.

Palabras clave: Centro gerontológico; contratación laboral; funciones específicas; déficit presupuestal.

\title{
LABOR PROBLEMS AT GERONTOLOGY CENTERS IN THE CITY OF MEDELLIN
}

\begin{abstract}
:
A large percentage of gerontology centers are constituted as non-profit organizations (NPO), and it is their nature to expand their corporate purpose under the criteria of providing altruistic basic services for the elderly in order to help them live out their last years with dignity, rather than to favor personal gain for those who engage in the activity. This aspect of operating as a non-profit organization drives many such centers to lack sufficient resources to develop their activities and fully cover all the needs of their users. Consequently, many of the duties to be fulfilled by professionals and experts responsible for the care and attention of the elderly are neither clearly stipulated nor covered by adequate employment contracts, as many time the duties of these practitioners merge with those of other assistants, thereby resulting in an inadequate provision of service. Just as hiring on an hourly basis or contracting for service provision or work denies them job stability, this forces practitioners to turn to other entities for additional employment, or to spend much of their time on other activities, which not only obfuscates the specialization of the services provided, but also the human sense of permanent care for the users. This situation then becomes one more obstacle in providing adequate services, as well as for stability and permanence at the gerontology centers.
\end{abstract}

Keywords: Gerontology center; employment contract; specific functions; budget deficit 


\section{QUESTÕES TRABALHISTAS NOS CENTROS DE GE- RONTOLOGIA NA CIDADE DE MEDELLÍN}

\section{RESUMO:}

Os centros de gerontologia, em grande porcentagem, são constituídos como Entidades Sem Fins Lucrativos (ESAL), cuja natureza é desenvolver seu objeto social sob o critério da prestação altruísta de serviços básicos aos idosos, para que estes adultos vivam seus últimos anos em condições de dignidade e não para favorecer o ganho pessoal daqueles que desenvolvem a atividade. O Trabalho sem fins lucrativos leva a que uma grande maioria desses centros não possuam recursos suficientes para desenvolver suas atividades e cobrir as necessidades dos seus usuários. Esta situação leva aos profissionais e especialistas encarregados dos cuidados dos idosos a não ter uma claridade nas suas funções nem condições trabalhistas adequadas, já que muitas vezes se sobrepõem suas funções às de outros trabalhadores, resultando numa prestação de serviços inadequada. Da mesma forma, a contratação por horas ou pela prestação de serviços lhes impede uma estabilidade no emprego, forçando-os a buscar contratos paralelos ou a dedicar seu tempo a outras atividades, com o qual perde-se não só a especialização dos serviços oferecidos, mas o sentido de cuidado humanizado permanente para os usuários. O exposto torna-se um obstáculo para prover uma adequada prestação de serviços e para a estabilidade e permanência dos centros de gerontologia.

Palavras-chave: Centros de gerontologia, condições de trabalho, funções específicas, déficit orçamentário

\section{INTRODUCCIÓN}

La investigación sobre las necesidades jurídicas, sociales y económicas de los centros gerontológicos en la ciudad de Medellín se realizó entre los años 2013 y 2015 ante la innegable realidad de que más del 10\% de la población del municipio es mayor de sesenta y cinco años, y que la proliferación de centros gerontológicos en la ciudad corresponde a esta situación social. Lo anterior hizo necesario indagar sobre la calidad de los servicios que reciben los adultos mayores en los centros gerontológicos constituidos como Entidades Sin Ánimo de Lucro (ESAL), con el fin de determinar la pertinencia de ofrecer alternativas a las dificultades que se presentan en la conformación y el desarrollo de este tipo de instituciones.

Para llevar a cabo dicha investigación, se adelantó una metodología mixta con énfasis en lo cuantitativo, ya que se buscaron los elementos fácticos que dieran cuenta de las actividades que se llevan a cabo en las instituciones, tomando como referente las condiciones de satisfacción de las perso- 
nas que laboran con estas. Esto llevó a observar que uno de los aspectos más relevantes que surgieron de la investigación es la problemática laboral, la cual está relacionada, fundamentalmente, al tipo de contratación que se adelanta en los centros gerontológicos frente a los profesionales y expertos que desarrollan actividades encaminadas a lograr el equilibrio físico y psicológico de los usuarios de los centros; problemática que está ligada a la observancia de si cumplen o no, de una manera correcta, las funciones que les corresponden en su calidad de expertos en un área de las ciencias médicas y/o sociales o si, por el contrario, sus funciones se ven desvirtuadas cuando tienen que asumir actividades que corresponden a otros profesionales o expertos, dado que, debido a los déficit económicos en los cuales se encuentran estas instituciones, su contratación puede no garantizar sus derechos laborales y llevar a que en sus actividades deban realizar funciones para las cuales no están capacitados.

Esta problemática se hizo evidente a través de la investigación, lo que llevó a plantear que deben tomarse pautas fundamentales para establecer un manual de funciones con el objetivo de orientar a los directivos de los centros, a los trabajadores, a los usuarios y sus familias acerca de cuáles son las condiciones laborales que deben imperar en los centros gerontológicos, y cuáles son las funciones que le deben ser asignadas a cada uno de los profesionales y expertos que laboran en este tipo de instituciones, esto con el propósito de alcanzar niveles de eficacia en la prestación de los servicios que garanticen a los adultos mayores una condición de vida digna una vez que lleguen a un centro gerontológico con la intención de permanecer en él.

\section{CONTRATACIÓN LABORAL EN LOS CENTROS GERON- TOLÓGICOS}

Los centros gerontológicos, como toda institución, bien sea sin ánimo de lucro (ESAL) o con ánimo de lucro, deben acogerse a los criterios de ley para la vinculación laboral de las personas que trabajarán en el logro del objeto social propuesto. Es por ello que, en un comienzo, podría decirse que lo ideal fuese que las personas que se vinculen con los centros lo hiciesen bajo la modalidad de contrato laboral indefinido o, cuando menos, contrato laboral a término definido. Lo cierto es que, dadas las condiciones económicas de muchos centros y las funciones específicas que prestan muchas de las personas que tienen a su cargo el cuidado de los adultos mayores, los centros se ven forzados, en muchos casos, a realizar contrataciones especiales mediante la modalidad de prestación de servicios o contratos por obra o labor terminada, En consecuencia se hace necesario entender, en primer lugar, cuáles son las personas que realizan trabajos tendientes a prestar servicios a los adultos mayores en los centros especializados de cuidado, comúnmente denominados centro gerontológicos. 
Un aspecto relevante es que debe contarse con una planta administrativa compuesta, cuando menos, por un director y una secretaria, personas que deben estar vinculadas bajo la modalidad de contrato fijo, preferencialmente a término indefinido; sin embargo, en la institución se necesitarán otras personas para desarrollar labores permanentes, y que por tanto su contratación deberá hacerse bajo el mismo tipo de contrato laboral. Tal es el caso de los vigilantes y las personas que tienen a su cargo la alimentación y los oficios varios, dado que prestan una labor continua al servicio de la institución.

Además de estas personas que, dadas las características de la labor desempeñada, deberán tener una vinculación permanente con los centros, existe una serie de especialistas que deben atender a los usuarios en horarios específicos, y cuya contratación formal puede ocasionar situaciones deficitarias en muchas de estas instituciones que no cuentan con recursos suficientes, tal como se desprende de los resultados de la investigación que muestran que el $72 \%$ de las entidades encuestadas reciben en promedio menos de dos salarios mínimos mensuales por paciente atendido (Ver gráfico número 1), lo que es insuficiente para la atención integral del paciente, al tiempo que manifiestan que solo el $29 \%$ de ellos reciben ayuda alguna por parte de instituciones privadas y que ninguno de ellos recibe ayuda de los organismos gubernamentales, dado que en muchos entes territoriales tienen sus propios centros de atención estatal y que, además, la labor de los centros gerontológicos constituidos como ESAL es altruista y, por lo tanto, no cuentan con recursos propios que puedan permitir un desarrollo idóneo de las actividades en torno al cuidado, atención y manutención de los adultos mayores.

De estos especialistas, cuya labor es indispensable para una adecuada prestación de los servicios de los centros gerontológicos, cabe resaltar

\section{Gráfico Número 1.}

Ingresos mensuales a los centros gerontológicos por paciente atendido

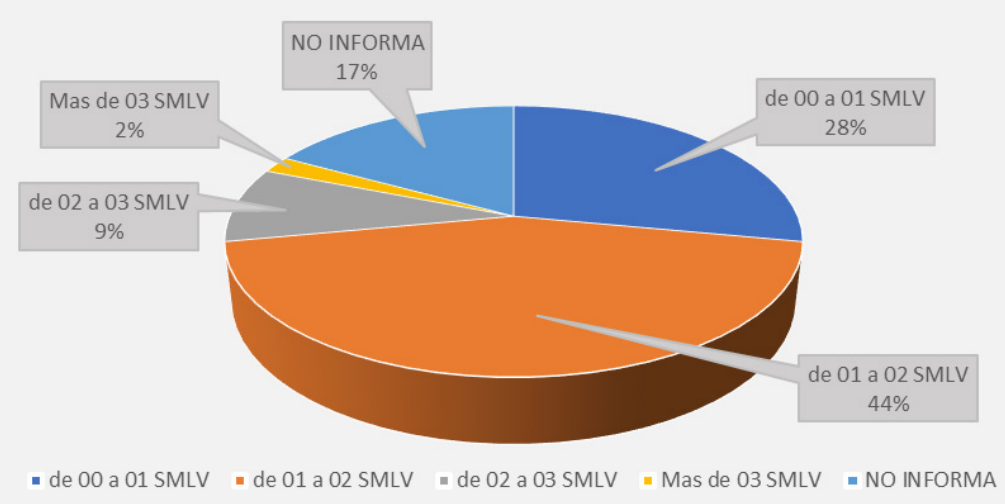


la labor de médicos, enfermeras, fisioterapeutas, recreacionistas, psicólogos, expertos en gerontología, terapia, respiratoria y odontología, por solo nombrar algunos fundamentales. Estos profesionales y expertos deberán ser contratados por las instituciones con el fin de que presten sus servicios, dado que de no mediar una relación laboral las demandas en contra de los centros podrían constituirse en un factor económico que llevaría al fracaso la destinación de los pocos recursos existentes.

Lo más idóneo sería que estos profesionales y expertos fuesen contratados a término indefinido o, cuando menos a término fijo, con el propósito de garantizar no solo su estabilidad laboral sino la prestación permanente de los servicios a los usuarios; sin embargo, debido al alto precio económico que esto representa y que, obligatoriamente, debe sumarse a los costos fijos y variables como arriendo, servicios públicos, alimentación y medicamentos, entre otros, se ha convertido en una costumbre la contratación de forma, si no totalmente irregular, sí mediante contratos por prestación de servicios o por obra o labor, con lo cual la estabilidad laboral de quienes prestan servicios en los centros es muy inestable debido que, según información suministrada por los centros gerontológicos encuestados solo el $9 \%$ de las personas que laboran en dichas instituciones se ve altamente vulnerada, sobre todo cuando al ser consultados sobre la vinculación a la seguridad social de las personas que laboran con los centros el $9 \%$ están afiliadas de manera formal a la seguridad social (Ver Gráfico número 2) con lo cual se pone en peligro, no solo la estabilidad

\section{Gráfico número 2}

Porcentaje personal de los centros gerontológicos vinculados a la Seguridad Social

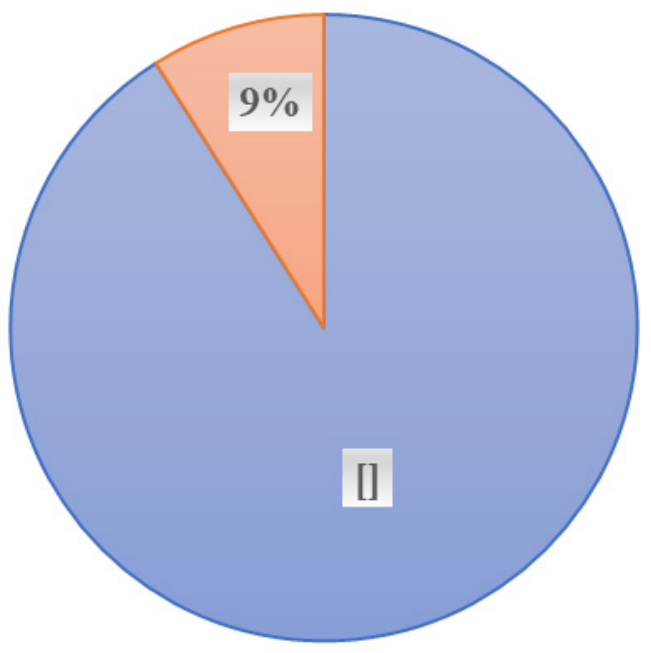


económica de los trabajadores y sus familias sino el funcionamiento mismo de los centros que pueden verse abocados a denuncias frente al no pago oportuno de los aportes de ley.

Si bien este margen está indicando que hay una preocupación por parte de las autoridades de los centros en la formalización de las personas que trabajan en las instituciones, no deja de ser preocupante, dado que este es un ítem en el cual debe existir una cobertura universal, puesto que de la no formalización de la planta laboral se desprenden obligatoriamente consecuencias funestas para el desarrollo económico de la institución que incumple sus obligaciones laborales, consecuencias tan funestas que pueden llevar incluso al cierre del establecimiento. Este es un punto neurálgico al cual debe prestársele mucha atención.

Esta situación ha llevado a que se presente un nivel de atención deficiente de estos servicios en muchos de los centros gerontológicos, dado que la contratación de las labores que prestan estos profesionales y expertos depende de la capacidad económica del centro, por lo tanto, por solo citar algunos de estos rangos de desatención, se debe tener presente las falencias que se hacen evidentes en los resultados de la investigación como podrá observarse de los datos suministrados por los centros encuestados, tomando solo algunos rangos que brinden una ilustración sobre el tema, como podría ser, a manera de ejemplo, falta de contratación de un equipo permanente de recreacionistas (Ver gráfico número 3)encargados de procurar un disfrute sano a estas personas que cuentan con todo el tiempo libre y para las cuales el esparcimiento se convierte en un factor fundamental para alcanzar un equilibrio físico y mental.

Este es un aspecto a mejorar debido a que la recreación es uno de los aspectos más necesarios en el equilibrio mental y en la recuperación anímica de los usuarios. Si a los inconvenientes propios de la vivencia en un centro gerontológico se le suman aspectos relacionados con el tedio y la falta de diversión, que es fundamental sobre todo a la gran cantidad de tiempo del cual disponen los usuarios, se pueden presentar episodios depresivos y otro tipo de traumas de orden psicológico que irían en detrimento de los adultos que residen en el lugar.

En este mismo rango de importancia debe colocarse la atención en fisioterapia para los usuarios de los centros debido a que las terapias para la recuperación física son indispensables cuando se piensa en una atención integral. El hecho de que solo la cuarta parte de los centros tengan terapeuta permanente es un indicio de una mala atención, sobre todo cuando cerca el $45 \%$ de las instituciones reconocen la falta total de prestación de este servicio (Ver gráfico número 4) que, junto con la atención médica, psicológica y de enfermería se constituye en uno de los ejes centrales de una vida digna en la institución. 


\section{Gráfico número 3}

Porcentaje de contratación de recreacionistas en los centros gerontológicos

$$
\text { ఐ Ocasional Permanente } ₫ \text { No hay }
$$

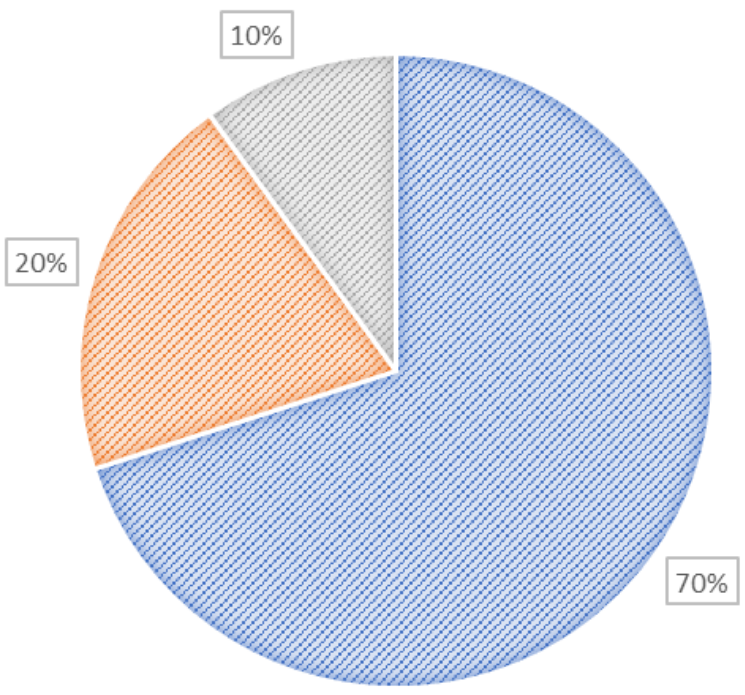

\section{Gráfico número 4}

Porcentaje de contratación fisioterapeutas en los centros gerontológicos

- Ocasional « Permanente No hay

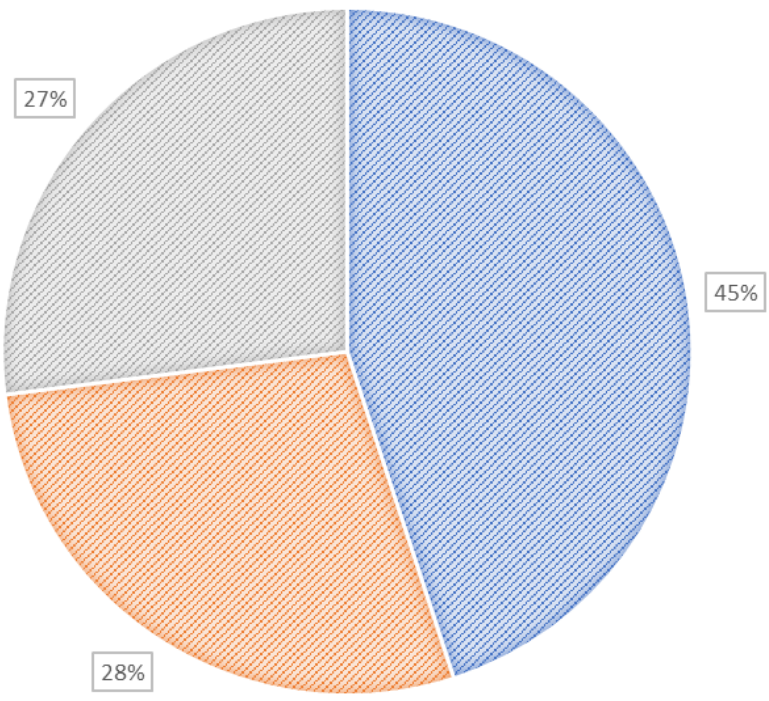


Otro aspecto que es preocupante hace relación a la cantidad y calidad del personal que es contratado por los centros gerontológicos en la ciudad de Medellín ya que, según datos derivados de la información suministrada por los directivos de dichos centros prácticamente uno de cada cuatro centros encuestados manifiesta carecer del personal suficiente para una adecuada atención de los usuarios (Ver gráfico número 5), lo cual es un indicativo de baja calidad en la prestación de los servicios. Este es un aspecto que debe ser mirado cuidadosamente puesto que influye directamente en la calidad de vida de los adultos mayores.

\section{Gráfico número 5}

¿La cantidad y calidad del personal es suficiente para una adecuada atención del Centro?

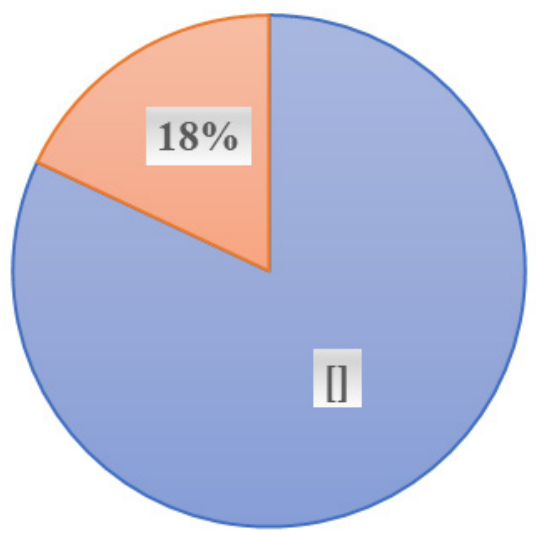

$\square S I \square N O$

Ante estas falencias que se desprenden de la información recolectada se hace evidente que la falta de personal permanente en las instituciones se constituye en uno de los mayores inconvenientes para un desarrollo adecuado de las actividades a realizar, lo cual redunda en la falta de calidad en la prestación de los servicios que se da a los usuarios, lo que hace necesario entrar a establecer si una adecuada contratación podría mejorar estos estándares de calidad en la atención.

Para ello es necesario definir las funciones que cumplen estos profesionales y expertos que prestan servicios complementarios a los administrativos y de atención médica permanente, con el fin de plantear la pertinencia de la contratación por prestación de servicios o, si por el contrario, los centros gerontológicos deberán hacer un mayor esfuerzo e incorporar a dichos profesionales y expertos como trabajadores vinculados a las instituciones bajo la modalidad de contrato de trabajo, ya sea a término fijo o a término indefinido. Con esto se lograría no solo la estabilidad laboral 
de estos profesionales sino también, asegurar la calidad en la prestación de los servicios.

\section{FUNCIONES DE LOS PROFESIONALES Y EXPERTOS AU- XILIARES EN LA ATENCIÓN DEL ADULTO MAYOR}

A pesar de que la norma establece que el manual de funciones es uno de los requisitos esenciales para establecer las condiciones mínimas en cuanto al manejo de personal en los centros gerontológicos (Resolución 8333 de 2004 de la Secretaría de Salud de Antioquia), no todos los centros lo tienen establecido puesto que el 16 por ciento de las instituciones encuestadas manifiestan no tener establecidos los manuales de funciones del personal (Ver gráfico número 6), lo cual genera un desorden que puede afectar la prestación de los servicios. Este es un aspecto en el cual debe buscarse una universalidad, dado que si los funcionarios tienen claras las funciones que deben cumplir, se alcanza un equilibrio en la prestación del servicio que redundará en beneficio de la atención a los usuarios.

\section{Gráfico número 6}

Porcentaje de centros gerontológicos que cuentan con un manual de funciones

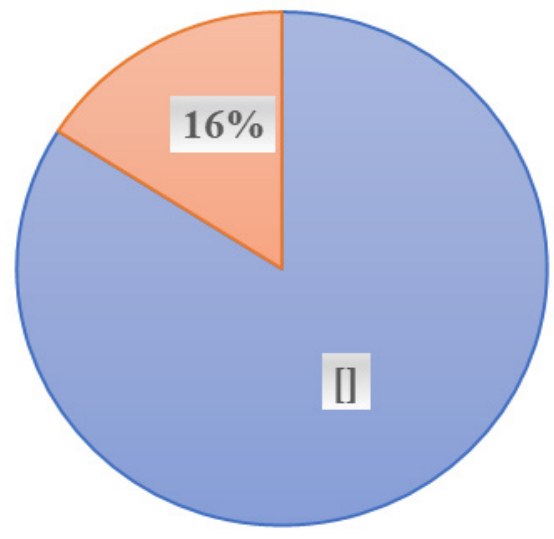

$\square \mathrm{SI} \square \mathrm{NO}$

Razón por la cual se hace necesario establecer claramente cuáles son las funciones que deben cumplir los profesionales y expertos auxiliares en los centros gerontológicos, con el fin de determinar la conveniencia o no, en términos de calidad, en la prestación de los servicios de una contratación formal en el campo laboral. Para esto se elaboraron en la investigación recuadros resaltando las principales funciones que deben cumplir dichos profesionales. 
A continuación se presenta un esquema genérico de cuáles deberán ser las funciones mínimas a cumplir por los diferentes servidores de los Centros gerontológicos; se advierte que no están incluidas todas las profesiones y oficios que podrían llegar a emplearse en dichas instituciones, y que las funciones que se presentan no son una obligación de ley sino simplemente un ejemplo que incluye, de manera general, las principales actividades que debe desarrollar cada una de las personas involucradas en el cuidado de los adultos mayores, siendo potestativo de las directivas de los centros aplicarlas en su totalidad, ampliarlas o restringirlas, conforme a las necesidades propias de cada institución.

\section{Cargo: médico}

NOMBRE DEL CENTRO GERONTOLÓGICO

MANUAL DE FUNCIONES PERSONAL OPERATIVO

\begin{tabular}{|l|l|}
\hline Cargo & Médico \\
\hline Área & Salud \\
\hline Nivel de estudios & Profesional \\
\hline Jefe inmediato & Director (a) del Centro. \\
\hline
\end{tabular}

\section{Funciones generales}

Deberá velar por la salud física de los usuarios y controlar los medicamentos y demás elementos que sean necesarios para el logro de este objetivo conforme a las políticas establecidas por el Centro.

\section{Funciones y tareas específicas}

1. Debe realizar el reconocimiento médico de los usuarios que llegan al Centro, elaborando la historia clínica y prescribiendo los tratamientos pertinentes para lograr su rehabilitación y prevenir que pueda desmejorarse su condición.

2. Informar a los familiares y demás interesados acerca del estado de salud de los adultos mayores que son atendidos en la Institución.

3. Participar con otros integrantes del área de la salud y demás involucrados en el proceso y en la programación de actividades grupales e individuales que contribuyan al mejoramiento general de los usuarios.

4. Atender tanto a los usuarios como al personal que pertenece al Centro, en caso de urgencia.

5. Si considera que el Centro no está en capacidad de atender en forma debida a un adulto mayor, deberá remitirlo inmediatamente a un centro hospitalario o de salud que esté habilitado para realizar el tratamiento. 
6. Deberá, además, supervisar las dietas alimenticias y los menús que se suministran a los pacientes, y preocuparse por las condiciones sanitarias del Centro, toda vez que estos son factores que tienen gran incidencia en la estabilidad de los pacientes.

Cargo: auxiliar de enfermería

NOMBRE DEL CENTRO GERONTOLÓGICO

MANUAL DE FUNCIONES PERSONAL OPERATIVO

\begin{tabular}{|l|l|}
\hline Cargo & Auxiliar de enfermería \\
\hline Área & Salud \\
\hline Nivel de estudios & Técnico profesional \\
\hline Jefe inmediato & Director (a) del Centro \\
\hline
\end{tabular}

\section{Funciones generales:}

Realizar las labores propias del cargo Auxiliar de enfermería mediante la prestación de los servicios primarios de salud a los usuarios, contribuyendo así con el objeto social de la organización.

\section{Funciones y tareas específicas:}

El Auxiliar de enfermería del área de salud deberá:

1. Atender y orientar a los usuarios de la institución de acuerdo con sus necesidades y expectativas, garantizando el cumplimiento de las políticas institucionales y normativas de salud.

2. Cumplir y velar con las normas de prevención relacionadas con infecciones nosocomiales que puedan presentarse al interior de la institución.

3. Suministrar los tratamientos médicos a los usuarios de acuerdo con la prescripción médica del caso.

4. Generar actividades y prácticas saludables en el ambiente de trabajo.

5. Trasladar a los usuarios a las citas médicas, exámenes y controles de salud en la institución requerida, según el nivel de atención.

6. Cuidar integralmente a los usuarios en condiciones críticas de salud, según su estado y de acuerdo con el criterio emitido por el médico.

7. Responder por la utilización adecuada del material, equipos y medicamentos al igual que controlar sus existencias.

8. Llevar en forma correcta y actualizada los registros (kardex y notas de enfermería) e historias clínicas de los pacientes. 
9. Asistir a las reuniones programadas por la institución.

10. Participar activamente en los procesos de cultura organizacional programados por las directivas del Centro.

11. Aplicar y mantener actualizados los procedimientos establecidos para el desarrollo de las actividades a su cargo.

12. Informar oportunamente a la Dirección de la institución las anomalías que se presenten en los servicios de enfermería.

13. Realizar baño asistido a los ancianos.

14. Prestar servicios de primeros auxilios a los ancianos que lo requieran.

15. Realizar control de los signos vitales a los ancianos.

16. Esterilizar, preparar y mantener en perfecto estado de limpieza el material, equipo y los elementos de enfermería, así como el sitio de trabajo.

17. Brindar educación en salud al anciano institucionalizado.

18. Ejercer las demás funciones que le sean asignadas por su jefe inmediato y que sean afines con las de su cargo.

Cargo: psicólogo

NOMBRE DEL CENTRO GERONTOLÓGICO

MANUAL DE FUNCIONES PERSONAL OPERATIVO

\begin{tabular}{|l|l|}
\hline Cargo & Psicólogo \\
\hline Área & Salud \\
\hline Nivel de estudios & Profesional \\
\hline Jefe inmediato & Director (a) del Centro. \\
\hline
\end{tabular}

\section{Funciones generales}

Propender por la estabilidad emocional y mental de los usuarios del Centro mediante la aplicación de técnicas y tratamientos que permitan su restablecimiento frente a desajustes propios de la edad y de las situaciones a las cuales pueda conllevar la internación en el Centro y el alejamiento de su entorno familiar.

\section{Funciones y tareas específicas}

1. Realizar una valoración de cada usuario al momento de ingresar al Centro, con el fin de establecer un diagnóstico de su salud física y mental, y así determinar los criterios de atención que se deberán seguir en cada caso particular.

2. Mantener actualizados los expedientes y las historias de vida de todos los pacientes a su cargo. 
3. Procurar el fomento de la autoestima y el autocuidado por parte de cada uno de los adultos mayores residentes en el Centro.

4. Atender de manera individual a cada uno de los usuarios que el equipo de trabajo considere que necesite una atención personal e individualizada.

5. Atender a los usuarios que de manera voluntaria se acerquen en busca de ser escuchados y orientados.

6. Hacer seguimiento permanente de todos y cada uno de los adultos mayores a su cargo.

7. Establecer políticas y criterios que minimicen los conflictos que puedan presentarse entre usuarios, acudientes, empleados y directivos.

8. Todas aquellas otras actividades relacionadas con su ejercicio que les sean asignadas por las directivas del Centro.

\section{Cargo: nutricionista}

NOMBRE DEL CENTRO GERONTOLÓGICO

MANUAL DE FUNCIONES PERSONAL OPERATIVO

\begin{tabular}{|l|l|}
\hline Cargo & Nutricionista \\
\hline Área & Salud \\
\hline Nivel de estudios & Profesional o técnico universitario \\
\hline Jefe inmediato & Director (a) del Centro \\
\hline
\end{tabular}

\section{Funciones generales}

Desempeñar labores profesionales como nutricionista para contribuir con el cumplimiento del objeto social del Centro, esto mediante la realización y el seguimiento de procesos y actividades comunitarias, clínicas y administrativas relacionadas con los diferentes niveles de atención, promoción, prevención, tratamiento y rehabilitación de los usuarios de la Institución.

\section{Funciones y tareas específicas}

El nutricionista del Centro deberá:

1. Aplicar las normas y procedimientos adoptados por el Centro en todos los campos relacionados con nutrición y alimentación.

2. Brindar atención nutricional a usuarios institucionalizados y en consulta ambulatoria y, así, promover hábitos de vida saludable.

3. Elaborar minutas de nutrición de acuerdo con el presupuesto establecido por el Centro y conforme a las diferentes patologías de los usuarios de la Institución. 
4. Evaluar y hacer seguimiento periódico a aquellas actividades relacionadas con el área de alimentación del Centro.

5. Presentar de forma periódica al superior inmediato un informe que contenga los resultados relacionados con el desarrollo de los programas de nutrición y del servicio de alimentación.

6. Planificar, organizar, dirigir, controlar y evaluar las actividades relacionadas con el servicio de alimentación mediante la implementación de medidas que permitan la capacitación del personal asignado a esta área.

7. Formular y presentar ante la Dirección del Centro un plan estratégico y uno operativo que tiendan hacia la optimización de los recursos disponibles para el área de Alimentación.

8. Realizar el seguimiento periódico a los planes estratégicos y operativos, previa aprobación de la Institución.

9. Participar de forma activa y propositiva en los procesos de cultura organizacional del Centro, especialmente en lo que concierne con procesos, riesgos, controles, calidad y documentación de la memoria institucional, de acuerdo con las directrices y parámetros impartidos por las instancias directivas.

10. Diligenciar de forma completa la historia nutricional de los usuarios de la Institución de acuerdo con los formatos establecidos y con letra legible

11. Aplicar y mantener actualizados los procedimientos establecidos para el desarrollo de las actividades a su cargo.

12. Ejercer las demás funciones que le sean asignadas por el jefe inmediato y sean afines con las funciones básicas y específicas.

Cargo: fisioterapeuta

NOMBRE DEL CENTRO GERONTOLÓGICO

MANUAL DE FUNCIONES PERSONAL OPERATIVO

\begin{tabular}{|l|l|}
\hline Cargo & Fisioterapeuta \\
\hline Área & Salud \\
\hline Nivel de estudios & Profesional o técnico universitario \\
\hline Jefe inmediato & Director (a) del Centro. \\
\hline
\end{tabular}

\section{Funciones generales}

El fisioterapeuta deberá establecer ciclos de ejercicios para lograr el proceso de rehabilitación de los usuarios procurando evitar que se presenten discapacidades y pérdidas funcionales, para esto utilizará equipamiento y ejercicios que permitan que se potencialice el sistema músculo esqueléti- 
co y que se minimicen las discapacidades de las personas atendidas conforme a las políticas establecidas por el Centro.

\section{Funciones y tareas específicas}

1. Definir y realizar los tratamientos que considere pertinentes para lograr la rehabilitación de los usuarios.

2. Colaborar con los otros integrantes del equipo del área de salud en la realización de pruebas y valoraciones relacionadas con su especialidad profesional.

3. Realizar un seguimiento permanente de los tratamientos aplicados y de la evolución de los pacientes.

4. Ejercer un control permanente de la evolución de los pacientes y de los tratamientos aplicados en aquellos eventos en los cuales el servicio se presta por fuera del Centro.

5. Actualizar su conocimiento frente a los recursos existentes en el ámbito territorial que puedan ser utilizados por la Institución para el mejoramiento del servicio prestado a los usuarios.

6. Participar activamente en los procesos interdisciplinarios que sean convocados por la Institución.

7. Informar a los familiares de los usuarios sobre los procesos y técnicas que deben aplicarse a los usuarios en el evento que se encuentren por fuera del Centro.

8. Prestar asesoría a los profesionales y funcionarios que deben tratar con los usuarios en aquellos aspectos que tengan que ver con el campo de las fisioterapias.

9. Participar en las reuniones institucionales en las cuales se haga evaluación y seguimiento de los procesos.

10. Otras actividades conexas que sean requeridas por las directivas del Centro.

Cargo: gerontólogo

NOMBRE DEL CENTRO GERONTOLÓGICO

MANUAL DE FUNCIONES PERSONAL OPERATIVO

\begin{tabular}{|l|l|}
\hline Cargo & Gerontólogo \\
\hline Área & Gerontología \\
\hline Nivel de estudios & Profesional o técnico universitario \\
\hline Jefe inmediato & Director (a) del Centro \\
\hline
\end{tabular}




\section{Función general}

Garantizar que el servicio y la atención suministrada al usuario adulto mayor sea acorde con su proceso de envejecimiento y vejez. La gerontóloga debe tener en cuenta que el usuario en su condición de adulto mayor presenta múltiples cambios que inciden en su comportamiento, de allí la importancia de ofrecer una atención integral (humana y profesional) que permita valorar la relación existente entre los aspectos funcionales, mentales y sociales como pilares de un conocimiento más amplio que garantice una mejor intervención.

\section{Funciones y tareas específicas}

1. Mantener al día toda la información de los usuarios con sus respectivos protocolos, en particular:
a. Ficha de ingreso
b. Ficha gerontológica
c. Ficha de autonomía gerontológica
d. Test Minimental
e. Evolución gerontológica

2. Además, el Gerontólogo debe cumplir con las siguientes funciones que contribuyen con el cumplimiento del objeto social del Centro:

a. Vigilar que la atención a los usuarios en salud, nutrición y acompañamiento psicológico sean adecuados y oportunos.

b. Controlar de forma periódica los signos vitales de los usuarios.

c. Suministrar los medicamentos prescritos.

d. Coordinar con la nutricionista que la alimentación que reciban los usuarios sea adecuada y balanceada.

e. Hacer seguimiento periódico a los cambios en el estado de ánimo de los usuarios.

3. Gestionar la documentación que requiera el usuario mediante la realización de los siguientes procesos:
a. Renovar cédulas.
b. Tramitar o renovar el SISBEN.
c. Colocar denuncias ante las instancias pertinentes.

4. Vigilar que el auto-cuidado diario de los usuarios sea adecuado por medio de actividades que conlleven hacia buenos procesos de:
a. Aseo personal
b. Vestuario
c. Ejercicio físico
d. Alimentación 
5. Coordinar a un equipo de trabajo que vele por la permanencia de un ambiente armónico, agradable e higiénico al interior del Centro a través de:

a. Aseo periódico y protocolizado en las habitaciones.

b. Garantizar el orden de las pertenencias.

c. Realizar sugerencias de decoración con colores agradables y llamativos.

d. Hacer recomendaciones para que las instalaciones cumplan con criterios como seguridad y firmeza.

6. Realizar un plan de trabajo que incorpore medidas periódicas que permitan conservar al máximo la autonomía tanto física como mental del adulto mayor mediante:

a. Actividades físicas (como caminatas y paseos).

b. Actividades lúdicas.

c. Terapia ocupacional.

d. Actividades que mejoren o estabilicen la parte cognitiva de los usuarios.

e. Charlas educativas.

\section{Cargo: ayudante de gerontología}

NOMBRE DEL CENTRO GERONTOLÓGICO

MANUAL DE FUNCIONES PERSONAL OPERATIVO

\begin{tabular}{|l|l|}
\hline Cargo & $\begin{array}{l}\text { Ayudante de gerontología o Cuidador de adul- } \\
\text { to mayor }\end{array}$ \\
\hline Área & Gerontología \\
\hline Nivel de estudios & Bachiller \\
\hline Jefe inmediato & Director (a) del Centro \\
\hline
\end{tabular}

\section{Función general:}

Prestar los servicios de atención personalizada a los usuarios en aquellas actividades en las cuales se requiere una ayuda personal como son la ayuda para levantarse de la cama, el aseo personal en aquellas actividades que no son posibles realizarlas de manera independiente por el usuario como el lavado de personas encamadas, el servicio de duchas, la afeitada y, en general, todas las actividades para las cuales el residente no está capacitado de una realización personal independiente.

Realizar las labores operativas propias de su cargo para contribuir al cumplimiento del objeto social del Centro mediante la prestación de servicios a los usuarios de la tercera edad. 


\section{Funciones y tareas específicas}

1. Desarrollar sus actividades de acuerdo con el cumplimiento estricto de los parámetros de vestuario exigidos para el porte de su uniforme (guantes, botas, etc.).

2. Tener a la mano los útiles de aseo personal necesarios para los usuarios: jabón, crema dental, enjuague bucal, cepillo de dientes y desodorante.

3. Tener disponible la ropa de cada usuario y fijarse que se encuentre en buen estado.

4. Disponer de los respectivos implementos de cama tales como: sábanas, fundas, toallas de baño y limpiones.

\section{Funciones y tareas particulares:}

Para aquellas funciones específicas en el cuidado de los usuarios deberá:

1. Trabajar en equipo.

2. Llevar bolsas negras grandes para depositar en estas los pañales desechables.

3. Bañar de forma independiente a cada usuario utilizando los implementos de aseo y paños para estregarlos; luego del baño sacarlos bien cubiertos con su toalla para vestirlos.

4. Garantizar que los usuarios entren al baño calzados.

5. Prestar la ayuda para que aquellos usuarios con discapacidades motrices cuenten con las herramientas necesarias (silla de ruedas, bastones, caminadores, etc.)

6. Recoger la ropa sucia, la cual debe salir clasificada de acuerdo con el siguiente protocolo:
a. Pijamas
b. Plásticos
c. Ropa interior
d. Sábanas y cobijas que hayan sido contaminadas con residuos fisiológicos deben ser puestas en el recipiente destinado para ello.

7. Sacar la ropa sucia a la lavandería.

8. Sacar las papeleras y llevarlas al lugar de las basuras.

9. Lavar los baños diariamente y desinfectarlos de acuerdo con la siguiente fórmula: Hipoclorito de sodio al $3.5 \%$ o $4 \%$ o a 500 partes por millón (p.p.m.); $10 \mathrm{~cm}$ cúbicos de hipoclorito por litro de agua; esta misma fórmula debe aplicarse para el lavado de los tapetes, traperos y limpiones.

10. Barrer, trapear, desinfectar y sacudir el salón. 
11. Guardar la ropa limpia que no se necesite en la zona de ropas del sótano.

12. Repartir la alimentación de media-mañana a las 10:30 A.M.

13. Llevar a los usuarios incontinentes al servicio sanitario cuando lo requieran.

14. Garantizar de forma frecuente el aseo de los usuarios incontinentes, así como su respectivo cambio de pañal.

Además, el personal responsable de la atención a los adultos mayores deberá:

a. Darle el alimento a los usuarios que tienen dificultades para comer por sí mismos.

b. Acostar a los usuarios al medio día, para su reposo.

c. Colocar cortinas oscuras en las ventanas para facilitar el descanso y, así, los usuarios puedan dormir.

d. Dejar a los usuarios no autónomos con su pijama puesta; fijarse que todos tengan pijamas y acostarlos.

e. Sacar las sillas de los baños para evitar accidentes.

f. Repartir vasos, platos y calzado a cada usuario.

g. Colaborar en la repartición de alimentos.

h. Brindar ayuda en el arreglo del comedor y el servicio de alimentación, de acuerdo con los protocolos establecidos por el Centro.

i. Traer los pocillos a cada salón para el tinto en la mañana.

j. Asistir a los programas de actualización programados por la institución.

k. Responder por la calidad en la prestación de sus servicios.

1. Conservar siempre los buenos hábitos y normas higiénico-sanitarias.

m. Responder por la conservación, mantenimiento y buen uso del material y equipo que tiene a su cargo.

n. Velar por la presentación personal de los usuarios.

o. Ejecutar las demás tareas que le sean asignadas por su jefe inmediato y sean afines con las funciones básicas establecidas.

NOTA: Las funciones 6-7-8-9-10-11 pueden ser asignadas al personal de oficios varios, en aras de la especialización de las funciones; no obstante, si el centro no cuenta con presupuesto suficiente deberán ser realizadas por el ayudante de gerontología. 
Cargo: trabajador social

NOMBRE DEL CENTRO GERONTOLÓGICO

MANUAL DE FUNCIONES PERSONAL OPERATIVO

\begin{tabular}{|l|l|}
\hline Cargo & Trabajador (a) Social \\
\hline Área & Social \\
\hline Nivel de estudios & Profesional o técnica universitaria \\
\hline Jefe inmediato & Director (a) del Centro \\
\hline
\end{tabular}

\section{Funciones generales}

Deberá planificar la programación de las actividades que permitan la integración de los usuarios con los demás internos en el Centro y velar para que el entorno en el cual se desarrollan las actividades, tanto grupales como individuales, sea el adecuado para una buena socialización de las relaciones conforme a los protocolos establecidos por la Institución.

\section{Funciones y tareas específicas:}

1. Planificar y ejecutar las actividades que permitan realizar estudios sobre la situación real de sociabilidad de los usuarios.

2. Adelantar las actividades administrativas y realizar los informes sobre los usuarios que sean solicitados por las directivas del Centro con el propósito de verificar la verdadera situación personal, familiar y social de los adultos mayores residentes en la Institución.

3. Establecer programas que permitan fomentar la integración y la participación de los usuarios en las actividades que se desarrollan en el Centro y en su entorno.

4. Crear y poner en marcha programas que permitan la integración social y la adaptación a la situación actual de las personas que son usuarias del Centro.

5. Coordinar los grupos de trabajo social y de aquellos encargados de participar en eventos de animación sociocultural.

6. Participar activamente en la comisión técnica que se cree para generar acciones de carácter social y colectivo.

7. Gestionar ante entidades e instituciones locales en caso de que se presenten eventos en los cuales los usuarios del Centro entren en conflicto con dichas instituciones.

8. Participar con el equipo del sector de la salud en la elaboración de las políticas de atención que se deberán prestar a los usuarios.

9. Participar en la reestructuración de los espacios que deberán utilizar los adultos mayores, tanto a nivel locativo como a nivel recreacional.

10. Visitar a los usuarios enfermos. 
11. Realizar otras funciones que le sean asignadas por las directivas del Centro y que estén directamente relacionadas con las actividades propias de su ejercicio profesional.

Cargo: gestor de actividades socio culturales NOMBRE DEL CENTRO GERONTOLÓGICO MANUAL DE FUNCIONES PERSONAL OPERATIVO

\begin{tabular}{|l|l|}
\hline Cargo & Gestor de actividades socio culturales \\
\hline Área & Social \\
\hline Nivel de estudios & Técnico o tecnólogo \\
\hline Jefe inmediato & Director (a) del Centro \\
\hline
\end{tabular}

\section{Funciones generales}

Velar para que los usuarios del Centro dispongan de actividades que les permitan recrearse bajo parámetros de seguridad, con la finalidad de lograr no solo el esparcimiento personal sino una efectiva socialización con los demás adultos mayores pertenecientes a la Institución, creando así un ambiente que permita mejorar la condición física y mental de los usuarios, todo ello conforme con las políticas establecidas para por las directivas del Centro.

\section{Funciones y tareas específicas}

1. Proponer y poner en ejecución programas encaminados a la gestión educativa de los usuarios.

2. Participar en la búsqueda de recursos económicos que permitan adelantar programas culturales y de educación.

3. Garantizar que las personas que participan en los eventos de orden cultural y educativo están capacitadas para el logro objetivo que se proponen dichas actividades.

4. Presentar evaluaciones presupuestales y de resultados de las actividades realizadas.

5. Realizar programas específicos que permitan adelantar líneas de acción con los adultos mayores.

6. Fomentar la educación integral de los usuarios mediante la realización de eventos.

7. Fomentar la realización de actividades individuales y grupales que posibiliten la ocupación del tiempo libre de las personas que residen en el Centro.

8. Generar políticas de motivación para la participación en las actividades programadas. 
9. Supervisar que no se presenten accidentes o situaciones que puedan poner en peligro a los usuarios y que sean derivadas de las actividades programadas.

10. Programar reuniones con los miembros de los equipos recreativos y con gestores de otros Centros con el objetivo de proponer y adelantar políticas conjuntas.

11. Fomentar la inclusión de un voluntariado en el Centro que permitan acrecentar las prácticas socioculturales.

12. Comunicar a las directivas de la Institución cualquier anomalía que pueda presentarse en el desarrollo de las funciones propias de su cargo.

13. Realizar todas aquellas actividades que le sean asignadas por las directivas del Centro y que tengan relación con su actividad profesional.

\section{Cargo: responsable de lavandería}

NOMBRE DEL CENTRO GERONTOLÓGICO

MANUAL DE FUNCIONES PERSONAL OPERATIVO

\begin{tabular}{|l|l|}
\hline Cargo & Responsable de lavandería \\
\hline Área & Servicios generales \\
\hline Nivel de estudios & No se requiere \\
\hline Jefe inmediato & Director (a) del Centro \\
\hline
\end{tabular}

\section{Funciones generales}

La persona responsable de lavandería realizará labores relacionadas con el proceso de lavado de ropa (que consiste en la clasificación y entrega de esta última a los usuarios); de esta forma, al desarrollar estas actividades operativas el personal contribuye con el cumplimiento del objeto social del Centro por medio de la prestación de un servicio integral y de calidad a los usuarios.

\section{Funciones y tareas específicas}

La persona responsable de lavandería deberá realizar las siguientes actividades:

1. Trabajar en equipo.

2. Recoger toda la ropa sucia de los usuarios para clasificarla de acuerdo con los protocolos establecidos. Para el cumplimiento de esta tarea la ropa no debe ser arrojada al piso, se debe utilizar los recipientes adecuados para esta función. 
3. La ropa disponible para el proceso de lavado debe ser clasificada de la siguiente forma:

a. Ropa de color (vestidos, tendidos de cama en algodón)

b. Ropa blanca (sábanas, toallas, trapitos, pijamas)

c. Cobijas.

4. Desmanchar la ropa disponible para el proceso de lavado antes de colocarla en la lavadora.

5. La dosificación para cada tanda de ropa debe ser la siguiente: agregar un pocillo de detergente (especialmente designado para la lavadora), en el momento indicado, durante el proceso de lavado se le agrega $1 / 3$ de blanqueador (a la ropa blanca) y $1 / 3$ de suavizante.

Así mismo, para aquellas funciones relacionadas con el área de lavandería deberá:

1. Lavar a mano en la poceta con jabón de barra: pantalones, camisas, sacos, baberos y medias.

2. Colocar en remojo con detergente los artículos plásticos, agregarles desinfectante y luego enjuagarlos; finalmente, colocarlos a secar.

3. Lavar los zapatos después de remojarlos con jabón y restregarlos con cepillo.

4. Llevar la ropa para el proceso de secado en el patio.

5. La secadora no se debe usar en tiempo soleado.

6. Después de usar la lavadora hacerle el aseo correspondiente; además, estar pendiente de que le hagan mantenimiento periódico.

7. Durante el proceso de lavado se puede encender el ventilador; no obstante, este debe apagarse al culminar el proceso de lavado.

8. Hacer aseo diario al sanitario, baño, pocetas, vestidores y demás espacios en donde se guardan los implementos de aseo.

9. Entregar diariamente en las horas de la tarde los residuos de útiles de aseo, así como los recipientes donde se ponen los detergentes a la funcionaria designada.

10. Mantener esta zona completamente limpia y desinfectada de acuerdo con los protocolos establecidos por el Centro, los cuales indican que la desinfección se debe hacer de acuerdo con la siguiente formula: Hipoclorito de sodio al 3.5\% o 4\%, 200 partes por millón (p.p.m.), 4 centímetros de hipoclorito por un litro de agua.

11. Recoger la ropa seca, separar aquella que se va a planchar de la que se va a doblar, y guardar la ropa en su respectivo lugar. La ropa debe estar marcada con el nombre completo del usuario.

12. Planchar la ropa diariamente.

13. Mantener los armarios bien organizados. 
Además, se debe velar por el cumplimiento de las siguientes funciones relacionadas con el área de lavandería:

a. Buscar la ropa de las personas en situación de discapacidad física o mental para el día siguiente.

b. Responder por la conservación, mantenimiento y buen uso del material y equipo que tiene a su cargo, es decir, la lavadora, la secadora y demás equipos a su cargo.

c. Responder por la calidad en la prestación de sus servicios.

d. Conservar siempre los buenos hábitos y normas higiénicosanitarias.

14. Ejecutar las demás tareas que le sean asignadas por su jefe inmediato de acuerdo con la afinidad de las funciones básicas establecidas.

\section{Cargo: responsable servicio de alimentación}

NOMBRE DEL CENTRO GERONTOLÓGICO

MANUAL DE FUNCIONES PERSONAL OPERATIVO

\begin{tabular}{|l|l|}
\hline Cargo & Responsable servicio de alimentación \\
\hline Área & Servicios generales \\
\hline Nivel de estudios & $\begin{array}{l}\text { Técnico profesional con certificado y carné ac- } \\
\text { tualizado de manipulación de alimentos emi- } \\
\text { tido por institución reconocida y autorizada } \\
\text { para tal efecto. }\end{array}$ \\
\hline Jefe inmediato & Director (a) del Centro. \\
\hline
\end{tabular}

\section{Funciones generales}

Desempeñar las funciones de preparación de alimentos de acuerdo con criterios normativos, técnicos y de dirección en los horarios establecidos por el Centro. Se sugiere los siguientes horarios para cada comida: desayuno (8AM-8:30AM), media mañana (10:30AM - 11AM), almuerzo (12M-12:30PM), refrigerio (2:30PM-3PM), cena (5PM-5:30PM) y merienda a necesidad (para aquellos usuarios con dieta especial de 7:30PM$8 \mathrm{PM})$.

\section{Funciones y tareas específicas:}

El responsable del servicio de alimentación deberá:

1. Informar de forma periódica a la Dirección acerca de los alimentos faltantes para la realización de la minuta patrón y el ciclo de menús establecidos.

2. Preparar los alimentos de acuerdo con los requerimientos normativos y lo establecido en la minuta patrón. 
3. Responder por la conservación y mantenimiento del equipo y la dotación que tiene a su cargo.

4. Responder por la calidad de la dosificación de los alimentos.

5. Responder por el buen uso de los equipos y la dotación dispuestos para la zona de alimentación.

6. Velar por la calidad en la prestación de los servicios.

7. Asistir, participar y poner en práctica las recomendaciones realizadas por la nutricionista en sus cursos periódicos de capacitación.

8. Conservar siempre los buenos hábitos y normas higiénicas.

9. Tener entera disposición para el aprendizaje de innovaciones tecnológicas actuales como almacenamiento de alimentos, manejo del reciclaje de residuos sólidos y líquidos, desinfección de equipos, planta física, alimentos conservación de estos.

10. Ejercer las demás funciones que le sean asignadas por el jefe inmediato, de acuerdo con la afinidad de las funciones básicas establecidas.

Cada centro, de acuerdo con sus necesidades y a su capacidad económica, deberá determinar la conveniencia de vincular o no a estos profesionales y expertos en las distintas áreas involucradas en la atención y cuidado de los adultos mayores, teniendo presente que una mayor estabilidad laboral redundará, necesariamente, en una mejor prestación de los servicios que requieren los adultos mayores que son la razón de ser de los centros gerontológicos.

\section{CONCLUSIONES}

La gran mayoría de los centros gerontológicos analizados tienen grandes dificultades en cubrir, de una manera adecuada, las necesidades básicas atinentes a la prestación de los servicios que requieren los adultos mayores. Si bien esto es una realidad innegable, se debe procurar que este déficit que se presenta en los renglones presupuestales no recaiga sobre la calidad en la formación y en la contratación laboral de los profesionales y expertos que deben llevar a cabo cada una de las actividades especializadas que requiere la prestación de los servicios indispensables para garantizar la estabilidad física y emocional de los usuarios de los centros gerontológicos.

Por esta razón, las directivas de dichas instituciones deben tener claro cuáles son las funciones que debe cumplir cada una de las personas que realizan labores en los centros, cuál es la regularidad y el tiempo de permanencia de cada uno de ellos dentro de la institución, con el fin de cualificar y cuantificar el equilibrio que debe existir entre el tiempo de labor que se realiza en la institución y las necesidades objetivas de los usuarios. Además, debe tener en cuenta que un trabajador con una relación laboral estable adquiere no solo un mayor sentido de pertenencia frente a la 
institución, sino que no tendrá la necesidad de hacer contrataciones paralelas a fin de adquirir los emolumentos necesarios para una supervivencia digna para él y su familia, logrando así que se dé una mejor prestación de los servicios a los usuarios, por tanto desembocará en beneficio de estos al tener a su favor la prestación de una actividad eficiente por parte de todas las estructuras administrativas y laborales que integran la institución.

Si se logra establecer una seguridad laboral y de seguridad social para los trabajadores habrá una correlativa mejoría en la prestación de los servicios, en consecuencia, se cumplirá la naturaleza social de los centros gerontológicos que, a través de su espíritu altruista, propugna porque las personas de la tercera edad o adultos mayores alcancen altos niveles de tranquilidad en la última etapa de su vida terrena.

Finalmente, debe hacerse visible que cada centro gerontológico tiene sus características especiales, por tanto, las recomendaciones que se derivan de la investigación realizada no constituyen una camisa de fuerza que obligue a las autoridades de las instituciones a seguir sus directrices de una manera estricta; no obstante, sirven, a guisa de ejemplo, para que los centros operantes en la actualidad o aquellos que se creen en el futuro tengan unos criterios de referencia que les permitan determinar los tipos de contratación más favorables a sus intereses económicos y que posibiliten una mejor prestación del servicio a los usuarios que busquen los servicios de la institución.

\section{REFERENCIAS}

Acosta, P., Adrián, R; Salazar S, \& Bernabé, V. (2015). Revista Caribeña de Ciencias Sociales. Obtenido de http://www.eumed.net/rev/caribe/2015/11/geriatricos.html

Andrews, G. (insertar mes, 2008). Los desafíos del proceso de envejecimiento en las sociedades de hoy y del futuro. Encuentro Latinoamericano y caribeño sobre personas de Edad. Serie Seminarios y Conferencias CEPAL.

Arango, V, \& Ruiz, I. Diagnóstico de los adultos mayores. Informe de la Fundación Saldarriaga Concha. Disponible en http://www.sdp. gov.co/sites/default/files/diag_adul_mayor.pdf

Bazo, M. (2015). Envejecimiento y Sociedad: una Perspectiva Internacional. (insertar ciudad). Edición Médica Panamericana.

DANE. (2015) Censo general.

Ministerio de Protección Social. (2007) Diagnóstico preliminar sobre personas mayores, dependencia y servicios sociales en Colombia. Bogotá. 